\title{
Transphenoidal Surgery : The Optimal Approach to Chordoid Gliomas of the Third Ventricle?
}

\author{
Rodrigo Carrasco-Moro, M.D., Inés Castro-Dufourny, M.D., Ph.D., ${ }^{2}$ Ruth Prieto, M.D., Ph.D., José M. Pascual, M.D., Ph.D. ${ }^{4}$ \\ Department of Neurosurgery, Ramón y Cajal University Hospital, Madrid, Spain \\ Department of Endocrinology, ${ }^{2}$ Sureste Hospital and Francisco de Vitoria University, Madrid, Spain \\ Department of Neurosurgery, ${ }^{3}$ Puerta de Hierro University Hospital, Madrid, Spain \\ Department of Neurosurgery, ${ }^{4}$ La Princesa University Hospital, Madrid, Spain
}

\section{To the Editor,}

We have read in detail the interesting article entitled, " $E x$ panded Endoscopic Transnasal Approach to the Chordoid Glioma of the Third Ventricle: The First Case Ever Reported", published in 2016 in the Journal of the Korean Neurosurgical Society ${ }^{5}$. Some years ago, we were involved in the treatment of a case of chordoid glioma (CG) of the third ventricle and became highly interested in the study of this very rare kind of tumor ${ }^{1)}$. Since the first description of this entity in 1998, barely over 100 CGs have been reported on in the scientific literature, mostly as single case reports. Nevertheless, the accurate number of "hidden" CGs included in those articles dealing with the results of specific surgical approaches for third ventricle tumors of a heterogeneous nature has not been investigated yet. In 2008, three groups of experts in the endoscopic transphenoidal approach published their results after using this surgical corridor to remove cystic lesions of the sellar region in a multicenter study which included a single case of $\mathrm{CG}^{3)}$. Driven by scientific curiosity, we contacted two of the authors of that paper (Professors Luigi M. Cavallo and John A. Jane Jr.) who kindly agreed to provide us with more detailed information about their patient.

He was a 48-year-old man with a one year history of declining vision and a recent episode of sudden, transitory loss of consciousness. The clinical examination revealed right-side optic atrophy and incomplete left-side homonymous hemianopsia; the endocrinological evaluation proved the presence of partial hypopituitarism, with conservation of both thyroid and posterior pituitary function. With a presumed diagnosis of craniopharyngioma, this third ventricular neoplasm found on the preoperative magnetic resonance imaging (MRI) (Fig. $1 \mathrm{~A}$ and B) was totally removed through an endoscopically assisted transphenoidal route. The pathological features of the lesion were consistent with the diagnosis of CG. Following surgery, visual symptoms persisted and the patient developed panhypopituitarism, diabetes insipidus and somnolence, a clinical picture quite similar to that observed in the patient described in the article by Zeinalizadeh et al. ${ }^{5)}$. In the latter report, however, no detailed information concerning the evolution of the patient's visual symptoms nor the account of his long-term follow-up clinical data are provided. Regrettably,

- Received : August 2, 2017 •Accepted : August 30, 2017

- Address for reprints : Rodrigo Carrasco-Moro, M.D.

Department of Neurosurgery, Ramón y Cajal University Hospital, Colmenar Rd, Km 9.100, Madrid 28034, Spain

Tel : +34 9133687 73, E-mail : rocamo@gmail.com

This is an Open Access article distributed under the terms of the Creative Commons Attribution Non-Commercial License (http://creativecommons.org/licenses/by-nc/4.0) which permits unrestricted non-commercial use, distribution, and reproduction in any medium, provided the original work is properly cited. 

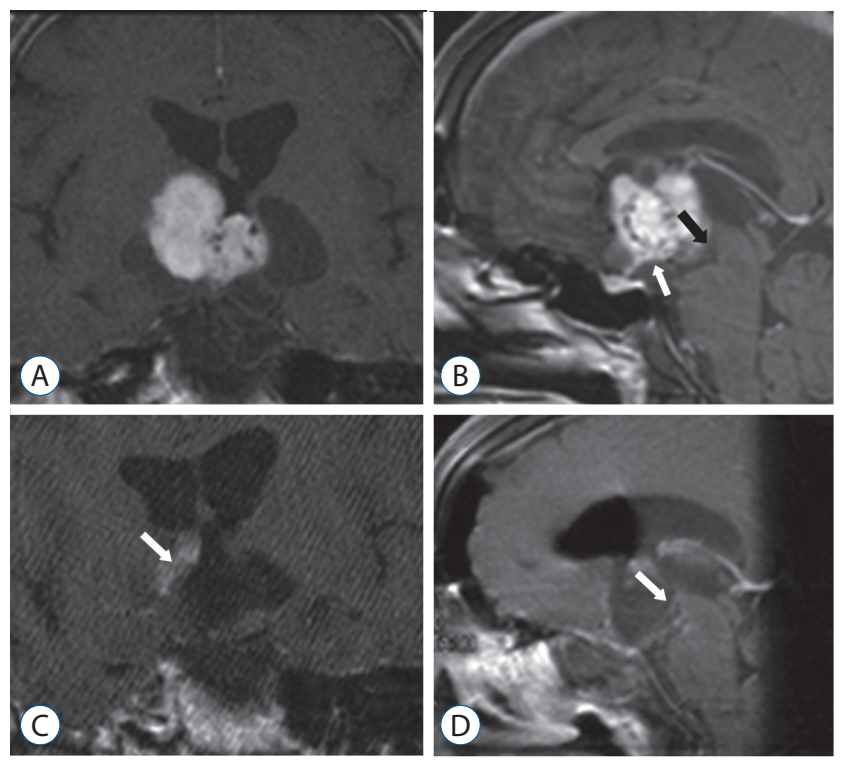

Fig. 1. A and B : Preoperative T1-weighted, contrast-enhanced MRI; coronal (A) and mid-sagittal (B) scans showing a well-defined, polylobulated, solid-cystic mass centered at the anterior third ventricular area, causing a displacement of the pituitary stalk (white arrow in B) and the mamillary bodies (black arrow in B). C and D : Postoperative T1weighted MRI-same sequence and scans- showing the anatomical defect at the region of the infundibulum, without identifiable median eminencepituitary stalk structures. Notice the presence of postsurgical changes involving the right IIIv wall (white arrow in C) and the atrophy of the decompressed mamillary bodies (white arrow in D). MRI : magnetic resonance imaging.

the patient reported by Cavallo et al. ${ }^{3)}$ was found dead in the rehabilitation unit several weeks after his surgical procedure, his sudden demise being thought the probable consequence of pulmonary thromboembolism.

The set of postoperative symptoms developed by both CG patients is consistent with the diagnosis of the infundibulotuberal syndrome together with a complete anterior pituitary insufficiency as a clinical expression of a hypothalamic-pituitary dysfunction. Indeed, in both patients the postoperative midsagittal MRI scans showed the same anatomical defect or "breach" at the third ventricle floor, spanning the median eminence and the proximal portion of the pituitary stalk (Fig 1C and D; and see Fig. 4 in reference 5). Such an anatomical defect should be considered the result of the surgical injury inflicted with the use of the transsphenoidal corridor chosen to remove the tumor, as CGs represent true third ventricle lesions which develop above an intact third ventricle floor.

CGs are usually described as pathologically benign, non-infiltrating neoplasms that, at least from a theoretical stand- point, could represent a model of a pure intraventricular mass that causes the displacement of the anatomical structures forming the boundaries of the anterior third ventricle, particularly the hypothalamus and the optic pathways, during its growth ${ }^{2}$. Therefore, the preoperative symptoms associated with CGs -which may include a variable combination of symptoms derived from hypothalamic dysfunction, including those specifically related to the dysfunction of the infundibulo-tuberal region- should alert practitioners, particularly neurosurgeons, to the strictly intra-third ventricle position of the tumor. In the case of CGs, these hypothalamic/infundibulotuberal disturbances are potentially reversible after tumoral debulking. Nevertheless, achieving the accurate radiological diagnosis of a CG preoperatively represents a fairly difficult task, given the very low incidence of these lesions and the lack of specific MRI signs. At least an exhaustive topographical assessment of every lesion growing around the anterior third ventricle should be carried out in order to confirm the integrity of the third ventricle boundaries, the essential information to plan the optimal surgical approach ${ }^{4)}$.

The optimal surgical approach to CGs has not yet been established and should be the subject of further studies including larger series of patients. Nevertheless, given the availability of third ventricle floor-sparing surgical alternatives which essentially include the trans-lamina terminalis, the transcallosal and the frontal-transcortical-transventricular corridors $^{1,2,4)}-$, the use of the transphenoidal approach is debatable in the specific case of CGs, as it seems to carry a higher risk of permanent pituitary and hypothalamic damage.

\section{CONFLICTS OF INTEREST}

No potential conflict of interest relevant to this article was reported.

\section{INFORMED CONSENT}

Informed consent was obtained from all individual participants included in this study. 


\section{- Acknowledgements}

We would like to express our gratitude to Prof. John A. Jane Jr. who kindly provided us with the clinical details of the case herein reported.

\section{References}

1. Carrasco R, Pascual JM, Reina T, Nieto S, Linera J, Sola RG : Chordoid glioma of the third ventricle attached to the optic chiasm. Successful removal through a trans-lamina terminalis approach. Clin Neurol Neu-

\section{rosurg $110: 828-833,2008$}

2. Castro-Dufourny I, Carrasco R, Pascual JM : Chordoid glioma: a new paradigm of hypothalamic dysfunction? Pituitary 20 : 393-394, 2017

3. Cavallo LM, Prevedello D, Esposito F, Laws ER Jr, Dusick JR, Messina A, et al. : The role of the endoscope in the transsphenoidal management of cystic lesions of the sellar region. Neurosurg Rev 31 : 55-64, 2008

4. Pascual JM, Prieto R, Carrasco R, Barrios L : Displacement of mammillary bodies by craniopharyngiomas involving the third ventricle: surgical-MRI correlations and use in topographical diagnosis. J Neurosurg 119 : 381-405, 2013

5. Zeinalizadeh M, Sadrehosseini SM, Tayebi Meybodi K, Sharifabadi AH : Expanded endoscopic transnasal approach to the chordoid glioma of the third ventricle : the first case ever reported. J Korean Neurosurg Soc 59 : 643-646, 2016 